\title{
Estudo do relacionamento ântero-posterior entre os arcos dentários decíduos, de crianças nipo-brasileiras, dos dois aos seis anos de idade
}

\author{
Dirce Yamada Kataoka*, Helio Scavone Jr.**, Flávio Vellini-Ferreira***, Flávio Augusto Cotrim-Ferreira**,

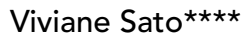

\section{Resumo}

Objetivo: verificar as prevalências dos diferentes tipos de relacionamentos ântero-posteriores entre os arcos dentários decíduos, bem como o possível dimorfismo sexual, em crianças nipo-brasileiras na faixa etária dos dois aos seis anos de idade. Metodologia: foram avaliadas por inspeção visual 310 crianças nipo-brasileiras, matriculadas em 19 escolas do estado de São Paulo, sendo 154 do gênero masculino e 156 do feminino. Os resultados foram submetidos ao teste do qui-quadrado e revelaram ausência de dimorfismo sexual para a maioria dos relacionamentos analisados, permitindo apresentar os resultados conjuntamente para ambos os gêneros. Resultados: as prevalências dos relacionamentos entre os segundos molares decíduos foram: $47,4 \%$ para o degrau mesial, $41,3 \%$ para o plano terminal reto, 5,5\% para o degrau distal e 5,8\% para os casos com assimetrias. Para os relacionamentos entre os caninos decíduos, conforme Foster e Hamilton, constataram-se prevalências de 77,4\% para a Classe 1, 6,8\% para a Classe 2, 6,8\% para a Classe 3 e 9\% para as relações assimétricas. Apenas a Classe 2 apresentou dimorfismo sexual, sendo de 3,9\% para o feminino e de 9,7\% para o masculino. Quanto à sobressaliência verificaram-se prevalências de $71,3 \%$ para a normal, 14,2\% para a aumentada, $7,4 \%$ para a mordida cruzada anterior e $7,1 \%$ para a mordida topo-a-topo. Resultados: concluiu-se que a maioria das crianças nipo-brasileiras avaliadas neste estudo, apresentaram um relacionamento ântero-posterior normal entre os arcos decíduos, merecendo atenção uma ligeira tendência para o desenvolvimento da Classe 3.

Palavras-chave: Dentição decídua. Oclusão dentária. Odontopediatria. Ortodontia.

\section{INTRODUÇÃO}

Para o estudo do relacionamento sagital entre os arcos dentários da maxila e da mandíbula (relação ântero-posterior), na dentadura decídua, é importante analisar o plano terminal entre os segundos molares decíduos, a relação entre os caninos decíduos, bem como a sobressaliência. $\mathrm{O}$ conhecimento das mudanças ântero-posteriores que ocorrem entre as dentaduras decídua, mista e permanente é muito importante para o clínico no

* Mestre em Ortodontia pela UNICID

** Professores Associados do Curso de Mestrado em Ortodontia da UNICID.

*** Coordenador do Curso de Mestrado em Ortodontia da UNICID.

**** Aluno do Curso de Mestrado em Ortodontia da UNICID. 
planejamento do tratamento ortodôntico interceptativo precoce.

As superfícies distais dos segundos molares decíduos guiam o local de erupção dos primeiros molares permanentes, enquanto as faces mesiais dos caninos decíduos orientam a localização e a disposição dos incisivos permanentes. Após isto, os caninos e os pré-molares irrompem no espaço limitado entre as faces mesiais dos primeiros molares permanentes e as faces distais dos incisivos laterais permanentes. Por sua vez, as faces distais dos primeiros molares permanentes orientam os segundos molares permanentes, completando-se o desenvolvimento da dentadura permanente e da oclusão $^{16}$.

O relacionamento ântero-posterior entre os arcos dentários decíduos pode ser influenciado pelos comportamentos psicológicos e sócio-culturais, como hábitos alimentares (aleitamento natural e artificial) e hábitos bucais não nutritivos (uso prolongado da mamadeira, sucção de chupeta e dedo).

Vários trabalhos, como os de Baume ${ }^{3}$; Farsi, Salama ${ }^{6}$; Infante ${ }^{10}$; Kerosuo ${ }^{13}$; Trottman, Elsbach ${ }^{24}$ e Woon ${ }^{26}$, relataram que existem diferenças no padrão oclusal, dependendo do grupo étnico estudado. Por esta razão, consideramos interessante realizar um estudo com crianças nipo-brasileiras para verificar as suas características oclusais.

Muito embora existam na literatura científica muitos trabalhos sobre o relacionamento ântero-posterior entre os arcos dentários decíduos ${ }^{1,7,14}$, dentre outros, não encontramos nenhuma pesquisa sobre este tema em crianças nipo-brasileiras.

Devido ao crescente número de descendentes japoneses que atualmente vivem no Brasil, acreditamos ser necessário um estudo mais individualizado para este grupo étnico.

\section{PROPOSIÇÃO}

Mediante a avaliação da dentadura decídua de crianças nipo-brasileiras na faixa etária dos 2 aos 6 anos de idade, este estudo propôs-se a determinar os índices de prevalência e o possivel dimorfismo sexual, para:

1) Os relacionamentos ântero-posteriores entre os segundos molares decíduos (plano terminal reto, degrau mesial, degrau distal e relações assimétricas);

2) As relações ântero-posteriores entre os caninos decíduos (Classes 1, 2, 3 e relações assimétricas) e;

3) A sobressaliência (normal, aumentada, topo-a-topo e mordida cruzada anterior).

\section{MATERIAL E MÉTODO}

Foram avaliadas 310 crianças nipo-brasileiras selecionadas em dezenove escolas de educação infantil, nas cidades de Bastos, Botucatu, Ibiúna, Marília, Mogi das Cruzes, São Paulo e Suzano, sendo 154 do gênero masculino e 156 do feminino, dos dois aos seis anos de idade.

Este estudo obedeceu a todos os preceitos e normas adotados pela Comissão de Ética em Pesquisa da Universidade Cidade de São Paulo e foi obtido por escrito, antes do início do estudo, o consentimento livre e esclarecido dos pais ou responsáveis.

As crianças avaliadas neste estudo atenderam aos seguintes critérios de inclusão:

- nascidas no Brasil e com no mínimo 50\% de ascendência japonesa direta, ou seja, a criança deveria possuir pelo menos um dos pais, dois avós ou quatro bisavós, maternos ou paternos, japoneses;

- com dentadura decídua completa, sem a presença de dentes permanentes erupcionados;

- sem cáries extensas ou grandes destruições coronárias que pudessem acarretar interferências nas relações oclusais;

- sem perdas precoces de dentes decíduos;

- sem anomalias dentárias de forma, número, estrutura e erupção e nunca submetidas a tratamento ortodôntico prévio.

Por meio do levantamento da ascendência das crianças, constatou-se que das 310 crianças nipobrasileiras avaliadas, 219 não eram miscigenadas, ou seja, possuíam 100\% de ascendência japonesa e 91 eram miscigenadas (mestiças). 


\section{Critérios para o exame clínico}

O exame clínico dos relacionamentos oclusais consistiu em inspeção visual, sendo realizado no próprio ambiente escolar, por três cirurgiãs-dentistas previamente calibradas, com a criança comodamente sentada e direcionada para uma fonte abundante de luz e com a oclusão em posição de máxima intercuspidação habitual (MIH).

Com auxílio de espátulas de madeira descartáveis, foram afastados os tecidos moles bucais para avaliação das relações ântero-posteriores dos segundos molares decíduos e caninos decíduos. A sobressaliência foi medida como a distância entre a superfície vestibular do incisivo central inferior e a borda incisal do incisivo central superior com os dentes em posição de máxima intercuspidação habitual. A medida foi quantificada usando-se uma régua milimetrada (Morelli), paralela ao plano oclusal.

Para a classificação dos relacionamentos entre as superfícies distais dos segundos molares decíduos foram utilizados os critérios propostos por Baume:

- plano terminal reto: quando as superfícies distais dos segundos molares decíduos estiverem coincidentes;

- degrau mesial: quando a superfície distal do segundo molar decíduo inferior estiver mais para mesial em relação à superfície distal do segundo molar decíduo superior;

- degrau distal: quando a superfície distal do segundo molar decíduo inferior estiver mais para distal em relação à superfície distal do superior;

Por sua vez, no relacionamento dos caninos foi utilizada a classificação de Foster e Hamilton ${ }^{8}$ :

- Classe 1: quando a ponta do canino decíduo superior estiver no mesmo plano da superfície distal do canino decíduo inferior em máxima intercuspidação habitual (MIH);

- Classe 2: quando a ponta do canino decíduo superior estiver numa relação anterior à superfície distal do canino decíduo inferior em MIH;

- Classe 3: quando a ponta do canino decíduo superior estiver numa relação posterior à superfí- cie distal do canino decíduo inferior em MIH.

Foram avaliadas também as relações mistas e assimétricas dos molares e caninos decíduos, ou seja, quando um dos lados não apresentava o mesmo tipo de relacionamento verificado no lado oposto.

A sobressaliência também foi classificada de acordo com os critérios de Foster e Hamilton ${ }^{8}$ :

- normal: quando a sobressaliência positiva não exceder a $2 \mathrm{~mm}$ (medida da superfície vestibular dos incisivos centrais decíduos inferiores até a borda incisal dos incisivos centrais decíduos superiores);

- aumentada: quando a sobressaliência positiva for maior que $2 \mathrm{~mm}$;

- topo-a-topo: quando as bordas incisais dos incisivos centrais decíduos superiores ocluírem com as bordas incisais dos incisivos centrais inferiores em posição de $\mathrm{MIH}$;

- mordida cruzada anterior: quando os incisivos centrais decíduos inferiores estiverem em relação anterior aos incisivos centrais decíduos superiores em posição de MIH.

\section{Análise estatística}

A análise estatística descritiva constou no cálculo, em termos absolutos e percentuais, das prevalências dos diversos tipos de relacionamentos oclusais ântero-posteriores entre os segundos molares decíduos, caninos decíduos e sobressaliência (trespasse horizontal entre os incisivos decíduos).

Efetuou-se a análise da significância estatística, mediante o teste do Qui-quadrado $\left(\chi^{2}\right)$, com nível de significância de 0,05 , para a verificação das possíveis diferenças entre os gêneros masculino e feminino quanto à prevalência destes diversos tipos de relacionamentos ântero-posteriores.

\section{RESULTADOS E DISCUSSÃO Relacionamento ântero-posterior entre os segundos molares decíduos}

A importância da relação entre as superfícies distais dos segundos molares decíduos ou plano 
terminal foi ressaltada por diversos autores ${ }^{2,15,16}$. A mudança do plano terminal reto para o degrau mesial é desejável para que o primeiro molar permanente erupcione em oclusão de Classe I de Angle (Fig. 1A). A persistência de um plano terminal reto conduz a uma oclusão cúspide a cúspide do primeiro molar permanente, que subseqüentemente poderá evoluir para um relacionamento de Classe I ou Classe II (Fig. 1B). Por outro lado, a presença de um degrau distal conduz ao estabelecimento da oclusão molar em Classe II (Fig. 1C).

$\mathrm{Na}$ tabela 1 e gráfico 1 podem ser observados os dados obtidos neste trabalho quanto à prevalência dos diversos tipos de relacionamentos ânteroposteriores entre os segundos molares decíduos, nos gêneros masculino e feminino, bem como na amostra total. Na tabela 1 visualizam-se os resultados da aplicação do teste do qui-quadrado para a avaliação do dimorfismo sexual destas características.

Como não houve diferenças estatisticamente significantes entre os gêneros para os diversos tipos de relacionamentos ântero-posteriores entre os segundos molares decíduos, os resultados foram avaliados num só grupo geral, demonstrando, dentro da classificação simetria, prevalências de $47,4 \%$ para o degrau mesial, $41,3 \%$ para o plano terminal reto e $5,5 \%$ para o degrau distal. A modalidade assimetria apresentou ocorrências de 4,2\%, 1,3\% e 0,3\% para as combinações respectivamente de plano terminal reto com o degrau mesial, plano terminal reto com degrau distal e degrau mesial com degrau distal.
Os relacionamentos entre os segundos molares decíduos em plano terminal reto e degrau mesial são considerados padrões normais para a dentadura decí$\mathrm{dua}^{2}$. O plano terminal reto se forma devido à maior largura mésio-distal do segundo molar inferior em relação ao seu antagonista superior. Por outro lado, quando os segundos molares decíduos apresentam o mesmo tamanho mésio-distal, suas faces distais formam um degrau mesial para a mandíbula.

Embora, trabalhos como os de Barbosa, Nicoló, Ursi ${ }^{1}$ e Ferreira et al. ${ }^{7}$ tenham constatado a relação em degrau mesial como a mais prevalente, a maioria dos trabalhos revisados neste estudo 2,6,8,9,14,22 apresentou uma maior prevalência para o plano terminal reto. Talvez, variações na metodologia e fatores étnicos tenham apresentado grande influência nestes valores.

Baume $^{3}$, em suas pesquisas nas ilhas do Pacífico Sul, concluiu que a distribuição da mésio e distoclusão tem tendências étnicas, ocorrendo a mesioclusão na maioria da população avaliada, sendo a distoclusão mais característica em descendentes de europeus.

Infante ${ }^{10}$, em um estudo sobre alguns aspectos da oclusão em crianças leucodermas, melanodermas e índias (apaches), observou uma grande prevalência da relação molar em Classe 2 nas crianças leucodermas, quando comparadas às crianças melanodermas e índias, em contraste com a relação em Classe 3, a qual apresentou uma alta prevalência entre as crianças melanodermas e índias.

Trottman e Elsbach ${ }^{24}$, realizando um estudo
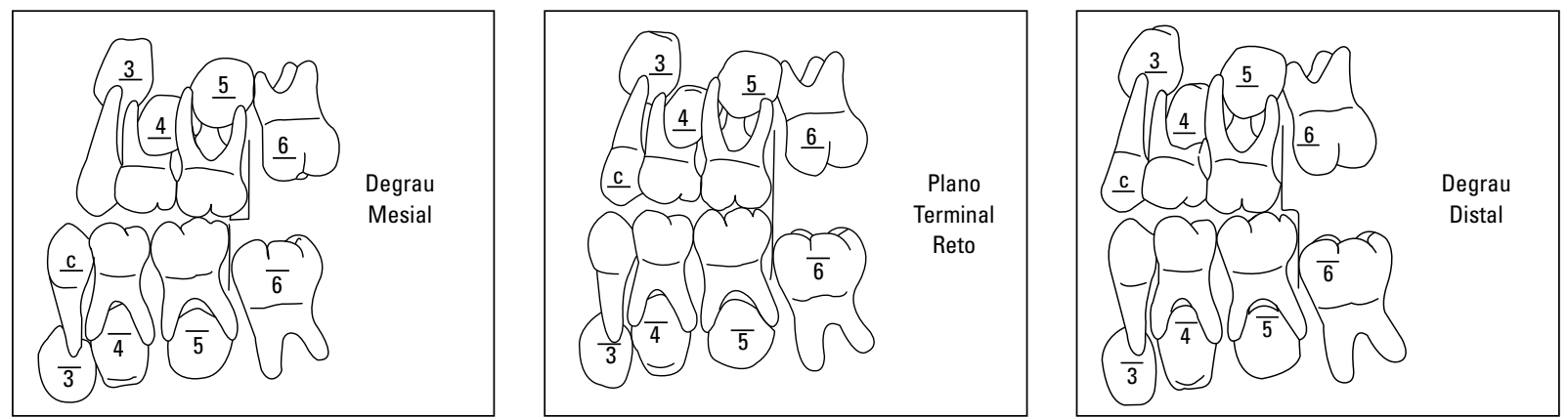

FIGURA 1 - Direção de erupção dos primeiros molares permanentes: A) Degrau mesial, B) Plano terminal reto, C) Degrau distal. 
sobre as más oclusões em crianças leucodermas e melanodermas, constataram uma significativa diferença étnica no padrão oclusal, sendo que a prevalência da relação molar de Classe 2 foi maior nas crianças leucodermas, enquanto a Classe 3 foi mais prevalente nas melanodermas.

Com relação ao degrau distal, Baume ${ }^{2}$ afirmou que este tipo de relacionamento oclusal é considerado indicativo de má oclusão na dentadura decídua, pois este não apresenta correção fisiológica espontânea e, caso o tratamento interceptativo não seja instituído na época adequada, o problema persistirá na dentadura permanente, caracterizando uma má oclusão de Classe II.

No presente estudo, observou-se uma baixa prevalência para este tipo de relacionamento $(5,5 \%)$, quando comparado com outros estudos revistos na literatura ${ }^{1,2,8,9,22}$. Esta baixa prevalência do degrau distal constitui um aspecto bastante favorável para a oclusão e pode estar relacionada a fatores étnicos e também culturais, tais como a pouca exposição a hábitos bucais deletérios.

A prevalência do degrau distal de 5,5\% na amostra total, verificada neste trabalho, foi quase semelhante àquela verificada no estudo de Isshiki et al. ${ }^{11}$, realizado no Japão com 121 crianças japonesas de 3 anos de idade, no qual constataram uma ocorrência de 4,8\% para este tipo de relacionamento entre os segundos molares decíduos.

Quanto aos relacionamentos assimétricos, neste trabalho observou-se uma ocorrência de 5,8\%, inferior ao resultado de Isshiki et al. ${ }^{11}$, que verifi-

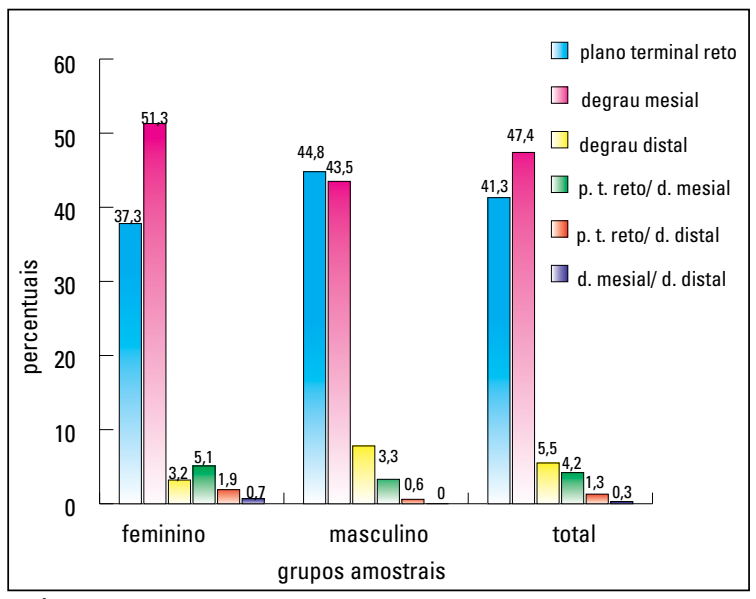

GRÁFICO 1 - Distribuição da amostra de crianças nipo-brasileiras, de acordo com os tipos de relacionamentos ântero-posteriores, simétricos e assimétricos, entre os planos distais dos segundos molares decíduos.

Tabela 1 - Distribuição da amostra e avaliação estatística do dimorfismo sexual em crianças nipo-brasileiras, de acordo com os tipos de relacionamentos ântero-posteriores, simétricos e assimétricos, entre os planos distais dos segundos molares decíduos.

\begin{tabular}{|c|c|c|c|c|c|c|c|c|}
\hline \multirow{2}{*}{ tipo } & \multicolumn{2}{|c|}{ feminino } & \multicolumn{2}{|c|}{ masculino } & \multicolumn{2}{|c|}{ total } & \multicolumn{2}{|c|}{ significância } \\
\hline & $\mathrm{n}$ & $\%$ & $n$ & $\%$ & $\mathrm{n}$ & $\%$ & $\chi^{2}$ & \\
\hline \multicolumn{9}{|l|}{ simétricos } \\
\hline plano terminal reto & 59 & 37,8 & 69 & 44,8 & 128 & 41,3 & 1,56 & n.s. \\
\hline degrau mesial & 80 & 51,3 & 67 & 43,5 & 147 & 47,4 & 1,88 & n.s. \\
\hline \multirow[t]{2}{*}{ degrau distal } & 5 & 3,2 & 12 & 7,8 & 17 & 5,5 & 3,15 & n.s. \\
\hline & 144 & 92,3 & 148 & 96,1 & 292 & 94,2 & & \\
\hline \multicolumn{9}{|l|}{ assimétricos } \\
\hline $\begin{array}{c}\text { plano terminal reto / } \\
\text { degrau mesial }\end{array}$ & 8 & 5,1 & 5 & 3,3 & 13 & 4,2 & 0,68 & n.s. \\
\hline $\begin{array}{c}\text { plano terminal reto / } \\
\text { degrau distal }\end{array}$ & 3 & 1,9 & 1 & 0,6 & 4 & 1,3 & 0,99 & n.s. \\
\hline \multirow[t]{2}{*}{$\begin{array}{l}\text { degrau mesial / } \\
\text { degrau distal }\end{array}$} & 1 & 0,7 & 0 & 0 & 1 & 0,3 & 0,99 & n.s. \\
\hline & 12 & 7,7 & 6 & 3,9 & 18 & 5,8 & & \\
\hline total & 156 & 100 & 154 & 100 & 310 & 100 & & \\
\hline
\end{tabular}

$\chi^{2}=3,84(p<0,05)$

n.s. $=$ não significante 
caram uma prevalência de $25 \%$ para as assimetrias. Este alto índice talvez se deva ao fato de que esse estudo baseou-se em modelos de gesso, nos quais pequenos desvios no relacionamento interdental tornam-se perceptíveis, gerando critérios de classificação muito mais rigorosos, elevando o número de casos com assimetrias.

Revelando um índice de prevalência abaixo dos resultados desta pesquisa, encontra-se o trabatho de Kaufman e Koyoumdjisky ${ }^{12}$, que avaliaram 313 crianças israelenses na faixa etária dos 3,5 aos 5,5 anos, constatando uma prevalência de 2,9\% de assimetrias nos relacionamentos entre os segundos molares decíduos, não esquecendo de mencionar que nesta pesquisa foram excluídas as crianças com má oclusão.

\section{Relacionamento ântero-posterior entre os caninos decíduos}

O relacionamento entre os caninos decíduos constitui um excelente dado auxiliar na classificação oclusal da dentadura decídua. Burstone ${ }^{4}$ ressaltou que em virtude do problema da topogênese dos primeiros molares permanentes, a relação terminal dos segundos molares decíduos não é o melhor guia para a avaliação da oclusão, sendo importante também avaliar a relação dos caninos.

$\mathrm{Na}$ tabela 2 e gráfico 2 podem ser observados os dados obtidos neste trabalho quanto à prevalência dos diversos tipos de relacionamentos ânteroposteriores entre os caninos decíduos, nos gêneros masculino e feminino, bem como na amostra total. Na tabela 2 visualizam-se os resultados da aplicação do teste do qui-quadrado para a avaliação do dimorfismo sexual destas características.

Verificou-se pela análise estatística que apenas

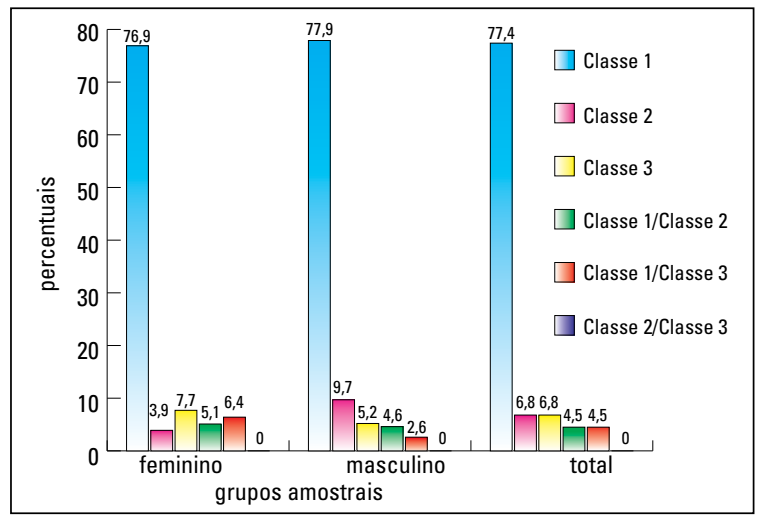

GRÁFICO 2 - Distribuição da amostra de crianças nipo-brasileiras, de acordo com os tipos de relacionamentos ântero-posteriores, simétricos e assimétricos, entre os caninos decíduos.

Tabela 2 - Distribuição da amostra e avaliação estatística do dimorfismo sexual em crianças nipo-brasileiras, de acordo com os tipos de relacionamentos ântero-posteriores, simétricos e assimétricos, entre os caninos decíduos.

\begin{tabular}{|c|c|c|c|c|c|c|c|c|}
\hline \multirow{2}{*}{ tipo } & \multicolumn{2}{|c|}{ feminino } & \multicolumn{2}{|c|}{ masculino } & \multicolumn{2}{|c|}{ total } & \multicolumn{2}{|c|}{ significância } \\
\hline & $n$ & $\%$ & $n$ & $\%$ & $n$ & $\%$ & $\chi^{2}$ & \\
\hline \multicolumn{9}{|l|}{ simétricos } \\
\hline Classe 1 & 120 & 76,9 & 120 & 77,9 & 240 & 77,4 & 0,04 & n.s. \\
\hline Classe 2 & 6 & 3,9 & 15 & 9,7 & 21 & 6,8 & 4,26 & 0,05 \\
\hline \multirow[t]{2}{*}{ Classe 3} & 12 & 7,7 & 8 & 5,2 & 21 & 6,8 & 0,80 & n.s. \\
\hline & 138 & 88,5 & 143 & 92,8 & 282 & 91 & & \\
\hline \multicolumn{9}{|l|}{ assimétricos } \\
\hline Classe 1 / Classe 2 & 8 & 5,1 & 7 & 4,6 & 14 & 4,5 & 0,06 & n.s. \\
\hline Classe 1 / Classe 3 & 10 & 6,4 & 4 & 2,6 & 14 & 4,5 & 2,61 & n.s. \\
\hline \multirow[t]{2}{*}{ Classe 2 / Classe 3} & 0 & 0 & 0 & 0 & 0 & 0 & 0 & n.s. \\
\hline & 18 & 11,5 & 11 & 7,2 & 28 & 9 & & \\
\hline total & 156 & 100 & 154 & 100 & 310 & 100 & & \\
\hline
\end{tabular}

$\chi^{2}=3,84(p<0,05)$

n.s. = não significante 
a Classe 2 apresentou dimorfismo sexual, sendo de 3,9\% para o gênero feminino e de $9,7 \%$ para o masculino. Os demais relacionamentos entre os caninos decíduos não apresentaram diferenças estatisticamente significantes, permitindo analisar os resultados conjuntamente para ambos os gêneros, revelando uma prevalência de $77,4 \%$ para a Classe 1 e $6,8 \%$ para a Classe 3. Nos relacionamentos assimétricos, as prevalências foram de $4,5 \%$ para a combinação de Classe 1 com Classe 2 e de 4,5\% para a combinação de Classe 1 com Classe 3.

Dado semelhante a este estudo quanto à prevalência do relacionamento entre os caninos em Classe 1, foi verificado no trabalho de Di Nicoló 5 (74,5\%). Prevalências mais altas, por volta de $85 \%$, foram verificadas nas pesquisas de Farsi, Salama ${ }^{6}$; Reyes, Sheen, Garcia-Godoy ${ }^{20}$, enquanto os trabalhos de Foster, Hamilton ${ }^{8}$ e Ferreira et al. ${ }^{7}$ demonstraram prevalências de $40 \%$ e $60 \%$, respectivamente.

Analisando a Classe 2, dentro da classificação simetria, observou-se dimorfismo sexual nas crianças, as quais apresentaram uma prevalência de 3,9\% para o gênero feminino e $9,7 \%$ para o masculino.

Considerando os resultados para a amostra total, a prevalência do relacionamento entre os caninos em Classe 2 verificada neste trabalho foi consideravelmente baixa $(6,8 \%)$, quando comparada aos outros estudos, cujos índices variaram de 10,9\% a $45 \% 5,6,8,18,23$. Talvez estas diferenças se devam a variações na metodologia e a fatores étnicos.

A prevalência da relação entre os caninos em Classe 3 de 6,8\% demonstrada neste estudo foi superior à verificada nos trabalhos de Farsi e Salama ${ }^{6}$ $(3,3 \%)$, Foster e Hamilton ${ }^{8}(1 \%)$, Otuyemi et al. ${ }^{18}$ (3\%) e Silva Filho et al. ${ }^{23}$ (2,93\%). Estas diferenças, conforme comentou-se anteriormente, talvez se devam a variações na metodologia e fatores étnicos.

Na modalidade assimetria, referente aos diversos tipos de relacionamentos ântero-posteriores entre os caninos decíduos, observaram-se prevalências de $4,5 \%$ para combinação de Classe 1 com a Classe 2 e de $4,5 \%$ para a combinação de Classe 1 com a Classe 3.
A mesma prevalência de 9\% para as relações assimétricas entre os caninos decíduos verificada neste trabalho, também foi encontrada na pesquisa de Otuyemi et al. ${ }^{18}$, em uma amostra de crianças nigerianas na faixa etária de 3 a 4 anos. Índice superior a este resultado encontra-se no estudo de Foster e Hamilton ${ }^{8}$ (14\%).

\section{Sobressaliência}

Para o estudo da relação ântero-posterior entre o arcos dentários decíduos é importante analisar o plano terminal entre os segundos molares decíduos, a relação entre os caninos, bem como a sobressaliência. A análise de todos estes fatores é importante para a avaliação da oclusão na dentadura decídua em desenvolvimento.

Na tabela 3 e gráfico 3 podem ser observados os dados obtidos neste trabalho quanto à prevalência dos diversos tipos de sobressaliência (trespasse horizontal entre os incisivos decíduos) nos gêneros masculino e feminino, bem como na amostra total. Na tabela 3 visualizam-se os resultados da aplicação do teste do qui-quadrado para a avaliação do dimorfismo sexual destas características.

Como não houve diferenças estatisticamente significantes entre os gêneros para os diversos tipos de sobressaliência, os resultados foram avaliados num só grupo geral, demonstrando que a sobressaliência normal foi a mais prevalente $(71,3 \%)$, seguida pela aumentada $(14,2 \%)$, depois pela mordida cruzada anterior $(7,4 \%)$ e, finalmente, pela mordida topo-a-topo $(7,1 \%)$.

Os resultados relatados, referentes à sobressaliência normal nos estudos de Farsi e Salama ${ }^{6}$ e Otuyemi et al. ${ }^{18}$, concordaram com os dados do presente estudo, cujas prevalências foram respectivamente de $76 \%$ e $68,8 \%$. Por outro lado, revelando índices de prevalências abaixo dos resultados desta pesquisa, encontram-se os trabalhos de Foster e Hamilton ${ }^{8}$ (25\%), Reyes, Sheen e GarciaGodoy $^{20}(53 \%)$ e Valente e Mussolino ${ }^{25}$ (55\%).

A prevalência da sobressaliência aumentada neste estudo $(14,2 \%)$ apresentou-se relativamente 
baixa, em relação ao estudo de Foster e Hamilton" ${ }^{8}$, que constatou um índice de $72 \%$ de trespasse horizontal aumentado. Por sua vez, com uma prevalência semelhante a este estudo, encontra-se o trabalho de Otuyemi et al. ${ }^{18}(14,7 \%)$.

A mordida topo-a-topo apresentou uma prevalência de $7,1 \%$, superior aos estudos de Foster e Hamilton $^{8}$ (2\%) e Valente e Mussolino ${ }^{25}$ (3,33\%). Porém, em relação ao estudo de Otuyemi et al. ${ }^{18}$ $(9,7 \%)$, esta apresentou-se menor.

A mordida cruzada anterior apresentou uma prevalência de 7,4\%. Este índice foi semelhante ao resultado observado no trabalho de Otuyemi et al. ${ }^{18}$ com crianças nigerianas de 3 a 4 anos de idade.

O estudo de Foster e Hamilton ${ }^{8}$, em crianças inglesas de 2,5 a 3 anos, revelou uma baixa prevalência para esta característica $(1 \%)$, denotando que talvez a mordida cruzada anterior tenha um forte componente étnico.

Um estudo comparativo efetuado em leucodermas, melanodermas e índios (Apaches), realizado por Infante ${ }^{10}$, demonstrou que os índios apresentaram uma maior prevalência de mordida cruzada anterior, seguidos pelos melanodermas e por último pelos leucodermas.

No trabalho de Trottman e Elsbach ${ }^{24}$, observou-se uma significativa diferença étnica na prevalência da mordida cruzada anterior, sendo de $16 \%$ para as crianças melanodermas e $4 \%$ para as leucodermas.

\section{Considerações finais}

Alterações ântero-posteriores, quando indicativas de má oclusão, devem ser diagnosticadas o mais precocemente possível, amenizando assim tratamentos futuros e proporcionando um bom crescimento e desenvolvimento do complexo craniofacial.

Com relação à futura oclusão na dentadura permanente, estudos longitudinais como os de Di Nicoló ${ }^{5}$ e Ravn ${ }^{19}$, dentre outros, demonstraram que a relação terminal dos segundos molares decíduos em degrau distal vai gerar invariavelmente uma relação de Classe II, enquanto as relações em

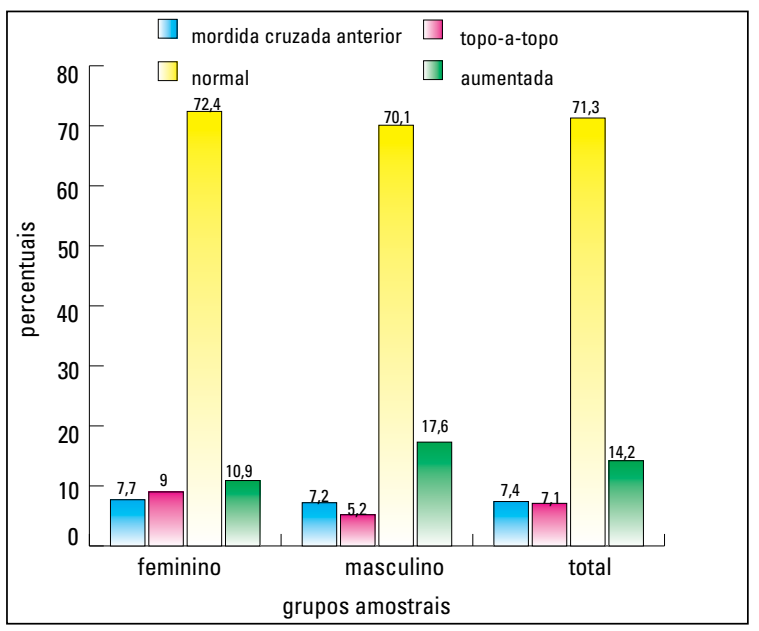

GRÁFICO 3 - Distribuição da amostra de crianças nipo-brasileiras, de acordo com os tipos de sobressaliência (trespasse horizontal entre os incisivos decíduos).

Tabela 3 - Distribuição da amostra e avaliação estatística do dimorfismo sexual em crianças nipo-brasileiras, de acordo com os tipos de sobressaliência (trespasse horizontal entre os incisivos decíduos).

\begin{tabular}{|c|c|c|c|c|c|c|c|c|}
\hline \multirow{2}{*}{ tipo } & \multicolumn{2}{|c|}{ feminino } & \multicolumn{2}{|c|}{ masculino } & \multicolumn{2}{|c|}{ total } & \multicolumn{2}{|c|}{ significância } \\
\hline & $\mathrm{n}$ & $\%$ & $\mathrm{n}$ & $\%$ & $\mathrm{n}$ & $\%$ & $\chi^{2}$ & \\
\hline $\begin{array}{c}\text { mordida cruzada } \\
\text { anterior }\end{array}$ & 12 & 7,7 & 11 & 7,2 & 23 & 7,4 & 0,03 & n.s. \\
\hline topo-a-topo & 14 & 9 & 8 & 5,2 & 22 & 7,1 & 1,68 & n.s. \\
\hline mormal & 113 & 72,4 & 108 & 70,1 & 221 & 71,3 & 0,20 & n.s. \\
\hline aumentada & 17 & 10,9 & 27 & 17,5 & 44 & 14,2 & 2,80 & n.s. \\
\hline total & 156 & 100 & 154 & 100 & 310 & 100 & & \\
\hline
\end{tabular}

$\chi^{2}=3,84(p<0,05)$

n.s. = não significante 
plano terminal reto ou degrau mesial geralmente resultarão, na maioria das vezes, em normoclusão (Classe I) e, em poucos casos, em má oclusão de Classe III na dentadura permanente.

Apesar da maioria das crianças deste estudo apresentar a relação entre os caninos em Classe 1 $(77,4 \%)$, as prevalências da Classe $3(6,8 \%)$ e da mordida cruzada anterior $(7,4 \%)$ foram relativamente altas, quando comparadas com outros estudos ${ }^{6,8}$. Devemos ficar atentos para o diagnóstico precoce destas desarmonias oclusais, para podermos reverter esta situação o mais cedo possível, evitando que influências negativas atuem sobre o crescimento normal da face.

\section{CONCLUSÕES}

Com base nos resultados alcançados nesta pesquisa e de acordo com a amostra e a metodologia empregadas, julgamos lícito concluir que:

1) As prevalências dos diversos tipos de relacionamentos ântero-posteriores entre os segundos molares decíduos não apresentaram dimorfismo sexual. Portanto, as taxas de prevalências foram associadas em um só grupo geral, conduzindo a índices de $47,4 \%$ para o degrau mesial, $41,3 \%$ para o plano terminal reto e $5,5 \%$ para o degrau distal, nas situações com simetria bilateral.
Além disso, nos casos com assimetrias foram verificados índices de prevalência de $4,2 \%$ para a combinação do plano terminal reto com o degrau mesial, 1,3\% para a associação do plano terminal reto com o degrau distal e $0,3 \%$ para a combinação do degrau mesial com o degrau distal.

2) Nos relacionamentos ântero-posteriores entre os caninos decíduos apenas a Classe 2 apresentou dimorfismo sexual, sendo, respectivamente, de $3,9 \%$ para o gênero feminino e $9,7 \%$ para o masculino. Como os demais relacionamentos não apresentaram diferenças estatisticamente significantes foram agrupados em um só grupo geral, conduzindo a índices de prevalência de 77,4\% para a Classe 1 e de $6,8 \%$ para a Classe 3 . Nos casos com assimetrias foram verificados índices de prevalências de $4,5 \%$ para a combinação Classe 1/Classe2 e de 4,5\% para a associação Classe 1/Classe 3.

3) Devido à ausência de dimorfismo sexual, as taxas referentes aos diversos tipos de sobressaliência foram agrupadas em um só grupo geral, conduzindo a índices de prevalência de 7,4\% para a mordida cruzada anterior, $7,1 \%$ para a mordida topo-a-topo, $71,3 \%$ para a sobressaliência normal e $14,2 \%$ para a sobressaliência aumentada.

Enviado em: outubro de 2004 Revisado e aceito: abril de 2005

\title{
Study of the anteroposterior relationship between deciduous dental arches of Japanese-Brazilian children, from 2 to 6 years of age
}

\begin{abstract}
Aim: the purpose of this cross-sectional study was to verify the prevalences of different types of anteroposterior relationships between deciduous dental arches and its possible sexual dimorphism, in Japanese-Brazilian children from 2 to 6 years of age. Methods: a total of 310 Japanese-Brazilian children from 19 schools in São Paulo, Brazil, comprising 154 males and 156 females, were examined by visual inspection. The qui-square statistical test was applied to evaluate the data, and the results revealed no statistically significant sexual dimorphism for the majority of types of anteroposterior relationship. Therefore, the results for both genders were grouped together. Results: the prevalences of anteroposterior relationships between deciduous second molars were: mesial step, $47.4 \%$; flush terminal plane, $41.3 \%$; distal step, $5.5 \%$ and asymmetric cases, $5.8 \%$. The prevalences of anteroposterior relationship of deciduous canines were: Class 1,77.4\%; Class 2, 6.8\%; Class 3, 6.8\% and asymmetric cases, 9\%. Only Class 2 revealed sexual dimorphism: $3.9 \%$ for female and $9.7 \%$ for male. The prevalences of overjet, were: normal, $71.3 \%$; increased, $14.2 \%$; anterior cross-bite, $7.4 \%$ and edge-to-edge bite, $7.1 \%$. Conclusion: it was concluded that the majority of Japanese-Brazilian children evaluated in this study showed normal anteriorposterior relationship between dental arches, with a slightly tendency for the development of Class 3.
\end{abstract}

Key words: Dentition primary. Dental occlusion. Pedodontics. Orthodontics. 


\section{REFERÊNCIAS}

1. BARBOSA, C. S.; Di NICOLO, R.; URSI, W. J. S. Estudo da prevalência dos tipos de planos terminais dos segundos molares decíduos. Pós-Grad Rev Fac Odontol São José dos Campos, São José dos Campos, v. 3, n. 1, p. 41-48, 2000.

2. BAUME, L. J. Physiological tooth migration and its significance for the development of occlusion: I. The biogenetical course of the deciduos dentition. J Dent Res, Washington, D.C., v. 29, no. 2, p.123-132, 1950.

3. BAUME, L. J. Uniform methods for the epidemiologic assessment of malocclusion. Am J Orthod, St. Louis, v. 66, no. 3, p. 251-272, 1974.

4. BURSTONE, C. J. Distinguishing development malocclusion from normal occlusion. Dent Clin North Am, Philadelphia, p. 479-491, 1964.

5. Di NICOLÓ, R. Estudo longitudinal das dentições decídua, mista e permanente, avaliando a relação molar, relação canina, sobremordida, sobressaliência e linha média. 1998. Tese (Doutorado)-Faculdade de Odontologia, Universidade de São Paulo, São Paulo, 1998.

6. FARSI, N. M. A.; SALAMA, F. S. Caracteristics of primary dentition occlusion in a group of Saudi children. Int J Paediatr Dent, Oxford, v. 6, no. 4, p. 253-259, 1996

7. FERREIRA, R. I.; BARREIRA, A. K.; SOARES, C. D.; ALVES, A. C. Prevalência de características da oclusão normal na dentição decídua. Pesqui Odontol Bras, São Paulo, v. 15, n. 1, p 23-28, 2001.

8. FOSTER, T. D.; HAMILTON, M. C. Occlusion in the primary dentition. Br Dent J, London, v. 126, p. 76-79, 1969.

9. GIMENEZ, C. M. M.; SIQUEIRA, V. C. V.; NEGREIROS, P. E. A importância da oclusão dos segundos molares decíduos sobre a dentadura permanente. J Bras Ortodon Ortop Facial, Curitiba, v. 5, n. 27, p. $67-75,2000$.

10. INFANTE, P. F. Malocclusion in the deciduous dentition in white, black and apache Indian children. Angle Orthod, Appleton, v. 45 , no. 3, p. 213-218, 1975

11. ISSHIKI, Y.; YAMAGUCHI, $H_{\text {.; }}$ YATABE, K.; KITAFUSA, Y.; KAWAMURA, M.; KANEMATSU, K. Longitudinal studies of dental models, especially on the occlusion of deciduous dentition of three year old children. J Tokyo Dent Coll, Tokyo, v. 70, no. 1, p. 113-129, 1969.

12. KAUFMAN, A.; KOYOUMDJISKY, E. Normal occlusal patterns in the deciduous dentition in preschool children in Israel. J Dent Res, Washington, D.C., v. 46, no. 3, p. 478-482, 1967.

13. KEROSUO, H. Occlusion in the primary and early mixed dentitions in a group of Tanzanian and Finnish children. J Dent Child, Chicago, v. 57, no. 4, p. 293-298, 1990.

14. MAGRI, V. C. Contribuição ao estudo epidemiológico do relacionamento ântero-posterior entre os segundos molares decíduos, dos quatro aos seis anos de idade. 2001. Tese (Mestrado)-Faculdade de Odontologia, Universidade Cidade de São Paulo, São Paulo, 2001.

15. MOYERS, R. E. Development of occlusion. Dent Clin North Am, Philadelphia, v. 13, no. 3, p. 523-536, 1969.

16. NAKATA, M.; WEI, S. H. Y. Guia de Oclusão em Odontopediatria. Tradução de Patrícia Resende. 1. ed. São Paulo: Ed. Santos, 1991.

17. NANDA, R. S.; KHAN, I.; ANAND, R. Age changes in the occlusal pattern of deciduous dentition. J Dent Res, Washington, D. C., v. 52, no. 2, p. 221-224, 1973.

18. OTUYEMI, O. D.; SOTE, E. O.; ISIEKWE, M. C.; JONES, S. P. Occlusal relationships and spacing or crowding of teeth in the dentitions of 3-4 year-old Nigerian children. Int J Paediatr Dent, Oxford, v. 7, no. 3, p. 155-160, 1997.

19. RAVN, J. J. Longitudinal study of occlusion in the primary dentition in 3 to 7 years old children. Scand J Dent Res, Washington, D. C., v. 88, no. 3, p. 165-170, 1980.

20. REYES, E.; SHEEN, J. E.; GARCÍA-GODOY, F. La occlusion de la dentición temporal en ninõs pre-escolares de Santo Domingo. Acta Odontol Pediatr, Santo Domingo, v. 1, no. 1, p. 11-22, 1980.
21. SERRA-NEGRA, J. M. C.; SERRA-NEGRA, E. Relação distal dos segundos molares decíduos. Arq Centro Est Curso Odontol, Belo Horizonte, v. 30, n. 1, p. 9-15, 1994.

22. SHIMIZU, R. H.; MICHAELIS, G.; LIU, J.; SHIMIZU, I. A.; IGNÁ$\mathrm{ClO}, \mathrm{A}$. S. Estudo das características da dentição decídua em crianças entre 3 e 6 anos de idade. J Bras Ortodon Ortop Facial, Curitiba, v. 8, n. 44, p. 124-131, 2003

23. SILVA FILHO, O. G.; SILVA, P. R. B. ; REGO, M. V. N. N.; SILVA, F. P. L.; CAVASSAN, A. O. Epidemiologia da má oclusão na dentadura decidua. Ortodontia, São Paulo, v. 35, n. 1, p. 22-33, 2002.

24. TROTTMAN, A.; ELSBACH, H. G. Comparison of malocclusion in preschool back and white children. Am J Orthod Dentofacial Orthop, St. Louis, v. 110, no. 1, p. 69-82, 1996

25. VALENTE, A.; MUSSOLINO, Z. M. Freqüência de sobressaliência, sobremordida e mordida aberta na dentição decídua. Rev Odontol Univ São Paulo, São Paulo, v. 3, n. 3, p. 402-407, 1989.

26. WOON, K. C. Primary dentition occlusion in Chinese, Indian and Malay groups in Malaysia. Aust Orthod, Brisbane, v. 10, no. 3, p. $183-185,1988$.

\section{Endereço para correspondência}

Dirce Yamada Kataoka

UNICID - Universidade Cidade de São Paulo

Rua Correia de Lemos, 501, apto 171 - Chácara Inglesa

CEP: 04.140-000 - São Paulo/SP

E-mail: dircekataoka@uol.com.br 